180 Dr. v. Linstow: Beitrag zur Anatomie von Phylline Hendorffii.

Fig. 15. Dotterzellen.

Fig. 16. Zellen der Schalendrüse.

Fig. 17. Ein Ei; a Keimzelle, b Dotterzellen, c Secret der Schalendrüse.

Fìg. 18. Längs-Sagittalschnitt, links von der Mittellinie; die Gefässe sind blau gezeichnet. a linke Kopfscheibe, b Schwanzscheibe, c Sehne, d Gefässerweiterung, e Vas deferens, $f$ Dotterdrüsen, $g$ linker Hoden.

Fig. 19-25. Querschnitte; die Nerven sind roth, die Gefässe blaı eingetragen.

Fig. 19. Durch die Kopfscheiben.

Fig. 20. Durch den Mund; a Cirrus, b Eiergang, c Dottergang.

Fig. 21. Durch das Ovarium; a Vas deferens, b Darm, c Bauch-, d Rückennerven.

Fig. 22. Durch die Hoden; a Vas deferens.

Fig. 23. Durch die Wurzel der Schwanzscheibe; a Rand, b Sehne, c Verstärkungslamelle.

Fig. 24. Durch die vorderen Haken der Schwanzscheibe; a Haken.

Fig. 25. Durch die mittlereu Haken der Schwauzscheibe; a Haken.

(Aus dem anatomischen Institut zu Breslau.)

Beiträge zur Kenntniss der Zelle und ihrer Theilung.

Von

\title{
Gustar PIatner.
}

\section{Die Entstehung and Bedeutung der Nebenkerne im Pan-} kreas, ein Beitrag zur Lehre von der Sekretion.

Hierzu Tafel XII.

In den folgenden Zeilen theile ich die Resultate von Untersuchungen mit, die mich schon seit längerer Zeit beschäftigen. Diese Beobachtungen sind von solch eigenartiger Beschaffenheit, dass man meine Bedenken, welche ich gegen eine allzurasche Publikation derselben trug, gerechtfertigt finden wird. Nachdem ich jedoch an einer grossen Anzahl von Amphibien und Reptilien übereinstimmende Befunde erhalten, nachdem ich sogar die hier im Pankreas sich abspielenden Vorgänge in ganz analoger Weise 
in den Malpighi'schen Gefässen der Insekten konstatirt hatte, habe ich es um so mehr für meine Pflicht gehalten, meinen früher an einigen Stellen gemachten Andeutangen hier eine ausführliche Beschreibung folgen zu lassen, als die Art und Weise, wie die fraglichen Elemente von Gaule und seinen Schülern zu weitgehenden Spekulationen benutzt werden, es erheischt, einmal eine sorgfältige Kritik zu üben. Eine abschliessende kann diese aber an dieser Stelle nicht werden, da ich zunächst noch einen andern Abschnitt zu behandeln habe, welcher von den regressiven Metamorphosen des Kerns und des Protoplasmas berichten wird. Das Durcheinanderwerfen progressiver und regressiver Prozesse hat es Gaule und seinen Anhängern möglich gemacht, eine Verwirrung anzurichten, in die es schwer hält Ordnung zu bringen. Zellparasiten in der Form von Hämatozoen und Coccidien spielen auch eine Rolle dabei (vergl. 1 und 8) und machen die Verwirrung noch grösser. Endlich ist die Einwanderung von Leukocyten noch ein Faktor, der in Betracht gezogen sein will. Die Aufgabe, welche ich mir stellte, als ich an die Bearbeitung dieses Themas herantrat, war demnach keine leichte. Zudem fielen meine ersten Beobachtungen in eine Zeit, wo unter dem Eindruck der epochemachenden Entdeckungen W. F l e m ming's gegen alle Art von Zelltheilung; welche nicht unter das von ihm aufgestellte Schema fiel, man nur zu leicht geneigt war, grosses Misstrauen zu hegen. In letzterer Beziehung ist erfreulicher Weise ein Umschwung eingetreten und auch von anderer Seite ist der Boden geebnet, auf dem ich mich zn bewegen habe und manches Hinderniss hinweggeräumt. Ich bin seit fünf Jahren mit dem Studium der Zelle und ihrer Bestandtheile beschäftigt und kann nichts anderes sagen, als wir sind noch weit entfernt von einer auch nur oberflächlichen Kenntniss derselben. Ich habe mich immer wieder von der Wahrheit des alten Satzes des Aristoteles uberzeugen müssen: "Man muss der Beobachtung mehr Glauben schenken als der Theorie, und dieser letztern nur dann glauben, wenn sie zu den gleichen Resultaten fưhrt wie die Erfahrung." Allen denen, welche im Schema ihr Heil suchen, möchte ich diesen Grundsatz des alten griechischen Forschers eindringlich vorhalten. Ich bin überzeugt, dass ich mit meinen Angaben vielfachen Widerspruch erfahren werde. Hier kann ich nur den einen Rath geben: nachzuuntersuchen und zwar an geeigneten Objekten, wie ich sie unten namhaft machen werde. 
Die besten und klarsten Resultate geben hier wieder die wirbellosen Thiere und doch werden diese bei derartigen Fragen mit einer Constanz vermieden, die Erstaunen erregt. Man quält sich mit den kleinsten Zellen, mit den schwierigsten Objekten, sieht einiges, übersieht das Meiste und stellt in die Lücken kühnt Combinationen, die dann das Fehlende ersetzen müssen.

Was nun zunächst meine Methode anlangt, so verwendete ich zur Härtung die oft bewährte stärkere Chrom-Osmium-Essigsäure-Mischung (Flemming), sowie Kleinenberg'sche Pikrinschwefelsäure 2. Th. mit Zusatz von Chromsäure. Gefürbt wurde mit Safranin, Hämatoxylin, Alauncarmin, Boraxcarmin, sowie endlich mit dem von mir als Kernschwarz (13) beschriebenen Farbstoffe. Ich gebrauchte stets nur eine Tinktion für sich. Die kombinirte Färbung, wie sie die Methode von Gaule erheischt, halte ich für völlig überflüssig.

Es kamen zur Untersuchung das Pankreas von I Chelonia: Emys europaea, Testudo graeca; II Sauria: Lacerta vivipara, Anguis tragilis; III Ophidia: Tropidonotus natrix, Coronella laevis. IV Anura: Rana esculenta, R. arvalis, R. fusca, Alytes obstetricans, Pelobates fuscus, Bombinator igneus, Bufo cinereus; V Urodela: Triton taeniatus, Salamandra maculata, Siredon pisciformis.

Bei sämmtlichen Thieren konnten die Nebenkerne in grösserer oder geringerer Anzahl nachgewiesen werden. Die grosse Zahl verschiedener Vertreter der meisten Klassen der Amphibien und Reptilien, welche ich durchforschte, gestattet demnach wohl den Schluss, dass die Nebenkerne im Pankreas von Amphibien und Reptilien ein regelmässiges Vorkommniss bilden. Die Malpigh ischen Gefässe von Hydrophilus piceus und Dytiscus marginalis zeigten analoge, wenn auch in einzelnen noch näher zu erörternden Punkten abweichende Verhältnisse. Bei ihnen sowie bei dem Salamander habe ich nun die Entstehung und Bedeutung der Nebenkerne näher untersucht. Die Gründe hierfür liegen in der ausgezeichneten Beschaffenheit der Zellen dieser Thiere für solche Zwecke. Die Zellen der Malpighi'schen Gefässe übertreffen die des Salamanders beinahe noch um das Vierfache, sind also ein ausgezeichnetes Objekt. Dabei bedürfen sie nur einer einfachen Vorbereitung, da man die Schläuche ohne weitere Zerkleinerung direkt untersuchen kann. Ihr Durchmesser fasst nur zwei Zellen. Man hat die letzteren also in toto vor sich, braucht nicht zu 
fürchten, dass durch das Schneiden Verletzungen entstehen oder wesentliche Theile der Beobachtung, indem sie auf verschiedene Schnitte fallen, entzogen werden.

Im Hungerzustande sind diese Zellen nabezu gleich gross. Sie zeigen einen hyalinen, ziemlich breiten Saum als äussere Begrenzung, der nur an der breiten Basis, wo sie der Drüsenwand aufsitzen, und an den Stellen, wo zwei benachbarte mit einander zusammenhängen, fehlt. Das Protoplasma ist von einem braunen körnigen Pigment durchsetzt, das um den grossen ovalen Kern unregelmässig angehäuft erscheint. Der letztere ist körnig, trägt ein oder mehrere Nukleolen and einige grössere Chromatinbrocken. Eingefasst wird derselbe von einer zarten Membran. Die nähern Details so wie die Art seiner Theilung habe ich in meinem vorigen Beitrag genauer beschrieben, worauf ich hier verweisen möchte. Die Sekretion wird nun damit eingeleitet, dass sich im respektive aus dem Protoplasma eine grössere Anzahl kugeliger Elemente, Sekrettropfen bilden. Die Zelle besitzt im Allgemeinen die Gestalt eines abgestumpften Kegels mit breiter Basis. Das obere Ende dieses Kegels wïrde dasjenige sein, wo dieser Prozess sich zuerst markirt. Diese Sekrettropfen entsprechen völlig den Elementen, die sich in den Leberzellen der Mollusken finden. Auf dieses besser bekannte Objekt, das auch ich oft gesehen habe, kann ich nich also bei meinen Vergleichen beziehen. Wie bei diesem tritt schliesslich ein Schwund der Kernmembran an dem in das Drisenlumen hineinragenden Theil der Zelle auf. Die Sekrettropfen kommen damit in dieses zu liegen.

Die Beschaffenheit der Sekrettropfen, hinsichtlich derer auch Frenzel $(5,6)$ für den Mitteldarm der Insekten genauere Angaben macht, ist nun folgende: Dieselben sind meist völlig kugelig, von wechselnder Grösse und glänzend. Kernfarbstoffe nehmen sie bis zu einem gewissen, jedoch wechselnden Grade etwas an. Im Innern erkennt man zuweilen eine netzförmige oder körnige Struktur, die ich aber für eine Reagentienwirkung zu halten geneigt bin. Diese Sekrettropfen gelangen nun dureh Schwand der Kernmembran in das Lumen der Drüse. Dass hierbei die ganze Zelle häufig zu Grunde geht, scheint sicher. Ich stimme also Frenzel bei, wenn er in ähnlicher Weise von den Zellen des Mitteldarms sich äussert: „Sie müssen demnach, wenn sie ihren Zweck erfüllen sollen, die 
von ihnen gebildeten und zur Verdauung nöthigen Stoffe ausscheiden.

Wie dies nun geschieht mag nicht in allen Fällen gleich sein. Wo aber mehr oder minder grosse, geformte und feste Sekretmassen sich angehäuft haben, was ja, wie wir gesehen haben, am häufigsten der Fall ist, können diese nicht anders frei werden, als indem sie den vor ihnen liegenden Theil der Zelle zerstören; and da nicht selten der ganze Zellleib dieht von ihnen angefiullt wird, wie bei vielen Käfern, Hymenopteren, Raupen u. s. w., so muss man zu dem Schluss gelangen, dass die ganze Zelle bei dieser Sekretion zu Grunde geht" (l. c. p. 301.) So ganz einfach ist nun aber der Verlauf der Sekretion nicht. Ausser dem Protoplasma betheiligt sich auch noch der Kern daran. Der Bildung der Sekrettropfen vorausgehend oder noch mit ihr zusammenfallend, bemerkt man eigenthlimliche Veränderungen an ihm, die auch Frenzel nicht entgangen sind und von ihm als „Kernsprossung“ (l. c. p. 181) bezeichnet werden. Er hat dieses bei Astacus beobachtet. Es ist mir sehr angenehm, wenigstens in der Feststellung einiger Punkte, hier einen Vorgänger zu haben. Dieses erwähnte sonderbare Pbänomen ist nun streng von der direkten Kerntheilung zu trennen. Das abgeschntirte Kernstiick bildet sich nicht zum selbständigen neuen Kern um, sondern wird ein Sekretstoff. Die direkte Kerntheilung, die auch von Frenzel genau beobachtet ist, führt meist zu zwei für das Augenmaass an Grösse gleichen Kernen. Die Kernsprossung - ich adoptire gern diesen Ausdruck Frenzel's führt hingegen zur Bildung des Nebenkerns.

Was nun die Bedeutung dieses Elementes anlangt, so könnte die von $\mathrm{Nuss}$ b a m gefundene Thatsache, dass derselbe besonders nach einer längern reichlichen Fütterung in der darauf folgenden Carenzzeit und zwar, wie der genannte Forscher mittheilt, etwa am vierten Tage sich reichlich findet, während längere Zeit hungernde Thiere ihn nur selten zeigen, zu der Ansicht verleiten, man habe es hier mit einer Erscheinung zu thun, welche der Ausfluss einer überreichen Ernährung der Thiere sei. Es wäre ja möglich, dass der Kern sich des ihm in Fülle dargebotenen und assimilirten Materials in dieser Form wieder entledige. Das Auftreten von sogar diffus im Kernsaft vertheiltem Chromatin spricht sehr für eine Vermehrung der specifischen Kernsubstanz. Eine solche findet also zweifellos statt. In wie weit die Nebenkern- 
bildung dazu dient, diesen Ueberfluss wieder zu eliminiren, darüber vermag jedoch das Experiment Aufschluss zu geben, welches $\mathrm{Og}$ a ta machte (12). Dieser zeigte, dass Pilocarpineinspritzung eine beträchtliche Vermehrung der Nebenkerne zur Folge hatte. Ich fand auch bei frisch eingefangenen Thieren oft eine grosse Menge Nebenkerne und bin daher der Meinung, dass man es hier mit einem Produkt der Sekretion zu thun hat, wenn ich auch anerkennen muss, dass weitere Beobachtungen und Experimente zur definitiven Feststellung dieser Sache noch recht erwünscht scheinen.

Nach seiner Abstossung vom Kern lässt sich unter den Sekretmassen der Mal pigh i'schen Gefässzellen der Nebenkern nur schlecht verfolgen, um so besser gelingt es im Pankreas. Da der Process der Kernsprossung jedoch hier genau gleicher Weise verläuft, wie in den Zellen der Malpighi'schen Gefässe, so können beide Objekte gemeinsam abgehandelt werden. In den zur Erläuterung dienenden Abbildungen habe ich nun mit peinlichster Genauigkeit unter steter Zubülfenahme des Prismas diese Vorgänge der Natur getreu zu kopiren mich bemüht, wie es die Wichtigkeit dieses Gegenstandes verlangt.

Der Nebenkern im Pankreas wurde bei Salamandra maculata, ebenso im Hepatopankreas von Astacus von N us s a um (10) entdeckt. Die historischen Data hierüber habe ich schon früher (13) zusammengestellt, worauf ich hier verweisen kann. Etwas später fand den Nebenkern $G$ a u l e (7) beim Frosch und andern Amphibien in demselben Organ. Meine ersten Beobachtungen darüber machte ich bei Anguis fragilis (13).

Die besten Bilder des Nebenkerns und seiner Bildung liefert unstreitig das Pankreas von Salamandra maculata. Der Axolotl steht auf gleicher Stufe, ist aber za kostspielig als Untersuchungsmaterial. Die Bildung des Nebenkerns kann ferner in ausgezeichneter Form an den grossen Kernen der Malpighi'schen Gefässe wahrgenommen werden.

Wenn hinsichtlich des Termins des Auftretens der Nebenkerne nach der letzten Fütterung ein Unterschied zwischen meinen Resultaten und denen $\mathrm{N}$ u s $\mathrm{b}$ a u m's sich ergeben hat, so ist folgender Umstand zu beachten. Ich habe meine Salamander stets mit Insekten, wie Käfern, Asseln, grossen Fliegen, denen ich die Fltigel abgeschnitten hatte, etc. gefüttert. Dieselben zeigten einen grossen Appetit und frassen soviel sie erhaschen konnten. Ich wollte ein- 
mal die naturlichen Bedingungen möglichst herstellen und sodann dachte ich mir, dass die schwer verdaulichen Chitinhüllen der genannten Kerfe vielleicht auch noch durch mechanische Reizung die Sekretion befördern könnten. Ich fand oft nach vier Tagen noch die genossene Nahrung zum Theil im Magen vor. Nussbaum hat, so viel ich aus persönlicher Mittheilung weiss, mit Fleisch seine Salamander gefuttert; das wird nattirlich rascher verdaut, worauf wohl seine kurzern Zeitangaben beruhen.

Ich beginne die Schilderung meiner Beobachtungen mit dem Stadium der erschöpften Drüsenzellen. Diese letzteren zeichnen sich aus durch ein sehr spärliches Protoplasma. Die äussern Contouren der Zelle sind unregelmässig. Die sonst so schön hervortretende regelmässige Anordnung in den Alveolen ist völlig verwischt. Auch der Kern zeigt eine wechselnde unregelmässige Form, er ist wie zusammengefallen mit Einbuchtungen und Höckern verseben. Das Nächste was an ihm zu bemerken ist, ist eine ausserordentliche Steigerung der Tinktionsfähigkeit des Kernsaftes, wodurch das Gerüstwerk undeutlich gemacht wird. Derartige Kerne leuchten in den mit Safranin gefärbten Präparaten mit dunkelrother Farbe hervor und zeichnen sich dadurch vor den andern Kernen, da stets die Alveolen verschiedene Stadien erkennen lassen, charakteristisch aus. Nach diesen unregelmässigen intensiv gefärbten und wegen des geringen Protoplasmas in Haufen nahe beisammenliegenden Kernen gilt es vor allen Dingen zu suchen, wenn man die Entwicklung des Nebenkerns beobachten will. An einem solchen Kern nimmt, während die andern Höcker verschwinden, eine Hervorragung alsbald eine besondere Form an. Das ganze im Kernsaft aufgestapelte Chromatin wandert in sie hinein, so dass sie als dunkelrothe Knospe dem mehr und melur zur normalen Beschaffenheit zurückkehrenden, das heisst einen unfärbbareu Kernsaft zeigenden tubrigen Theil des Kerns aufsitzt. Dieser Prozess findet sich genau in der gleichen Weise, wenn auch etwas kürzer, von Davidoff beschrieben für die Zellen des Darmepithels, scheint sich also auch hier zu finden (2).

Diese Knospe des Kerns hat eine mannigfaltige, von Zelle zu Zelle wechselnde Form. Sie ist grösser oder kleiner, bald einfach abgerundet, bald zwei- oder dreizackig, kleeblattförmig etc. Die Kernmembran überzieht dieselbe meist noch. Oft erscheint dieselbe aber auch geschwunden und der Inhalt der Kernsprosse 
liegt als fadiges oder gewundenes, oft zum Theil in Körner aufgelöstes, aber zunächst noch gefärbtes Element direkt im Protoplasma (Fig. 1, Taf. XII). Die Gerüstfäden des Kerns erscheinen in diesen Auswuchs wie hineingezehrt, so dass sie strahlig nach dem sich mehr und mehr einschnurenden Stiel desselben zusammenlaufen (Fig. 1 u. 7). Weiterhin trennt sich das sonderbare Gebilde vom Kern und verliert alsbald auch seine Tinktionsfähigkeit. Dies scheint nicht sofort in ganzer Ausdehnung zu geschehen. Ich habe solche Nebenkerne, wie man sie jetzt nenmen kann, zum Theil noch gefärbt, zum Theil blass gefunden (Fig. 4). Nur Hämatoxylin und Kernschwarz verleihen ihnen auch jetzt noch eine dunkle Färbung. Hand in Hand gehend mit diesen Veränderungen hat sich auch das Protoplasma der Zelle wieder vermehrt und gewinnt letztere ibr früheres Volumen allmählich wieder. Die beschriebenen Veränderungen des Kerns finden sich nie vereinzelt, sondern stets bei einer grössern Anzahl beisammenliegender Zellen zugleich, doch oft nicht genau auf demselben Stadium. Sehr schöne Bilder ergeben auch die Malpighi'schen Gefässe. Auch hier bemerkt man die wechselvollen Formen, die intensive Anhäufung von gelöstem Chromatin an diesen Elementen. Diese entwickeln sich hier meist nach dem in das Lumen der Drüse hineinragenden Ende der Zelle hin. Im Pankreas findet man die geschilderten Verhältnisse am bäufigsten gegen den 7. bis 8 . Tag nach der letzten Fütterung, selbstverständlich nur in einer beschränkten Anzahl von Alreolis, da die einzelnen Drüsenabschnitte beträchtliche zeitliche Schwankungen in ihrer Funktion und Entwicklung zeigen und anderseits das bescbriebene Phänomen rasch vorüber zu gehen scheint. Am 10. bis 11. Tage kann man sicher sein, nabezu in jeder Zelle einen auf diese Weise gebildeten Nebenkern zu treffen, da dieselben sich anch in den nicht völlig erschöpften Zellen entwickeln, andere solche sie von früherher noch besitzen.

Bei den Anuren und Reptilien wird die Beobachtnng dadurch erschwert, dass wegen des beschränkten Raumes in der Zelle die sich bildenden Nebenkerne sich kappenförmig dem Kern auflagern, so dass ich bei Anguis früher zu der Ansicht gelangen konnte, es seien durch Scheidewandbildung abgetrennte Kerntheile, doch gelang es mir auch hier das geschilderte Verhalten festzustellen.

Im ausgebildeten Zustande liegen nun die Nebenkerne stets 
in dem der Alveolenwand benachbarten Theil der Zelle, wie dies auch schon $\mathrm{Nussbaum}$ beschreibt. Die Zellen zeigen auf diesem Stadium etwas anter mittlerer Grösse. Zymogenkörnchen sind in ihnen nur spärlich oder gar nicht vorhanden. Die Form der Alveolen mit ibrem centralen Lumen fängt wieder an sich schärfer auszubilden. Die regressive Metamorphose des Nebenkerns und das stärkere Auftreten von Zymogenkörnchen schreiten nun gleichmässig fort. Bevor ich näher hierauf eingehe, möchte ich die Form und Zahl der Nebenkerne erst noch kurz besprechen. Meist findet sich in der Zelle nur eines dieser Elemente, häufig trifft man deren auch zwei, drei oder noch mehrere, die dann etwa zusammen dem Volumen eines einzelnen entsprechen. Sie sind gebaut wie der solitäre Nebenkern, blätterig oder lockig gewunden, breit oder schmal, abgerundet oder länglich, von wechselnden äusseren Umrissen, wie dies auch schon $\mathrm{Nussbaum}$ erkannt hat. Bei den Anuren and Reptilien sind die Nebenkerne meist balbmondförmig, der dem Drüsenlumen abgewendeten Seite des Kerns kappenförmig aufsitzend. Kernschwarz und Hämatoxylin verleilut ibnen insgemein eine dunkle Färbung (Fig. 8-10).

Mit dem stärkern Auftreten der Zymogenkörnchen in dem in Bezug auf den Alveolus centralen Theil der Zelle nimmt das Volumen der letzteren mebr und mehr zu. Die Tinktionsfähigkeit des Kerns wird eine geringere, er erscheint blass und nur die stärkern Chromatinkörner des Geriistes treten deutlich noch hervor. Auffallend ist im Gegensatz dazu die oft ziemlich intensive Färbung der Zymogenkörner mit Safranin. Der Nebenkern wird blasser bei Hämatoxylinfärbung, sein Volumen geringer; schliesslich scheint er ganz geschwunden und nur unter den Zymogenkörnern des centralen Theils nimmt man noch zuweilen einige fädige Reste desselben wahr. Er ist also in diesen Zelltheil hineingerïckt. Weiterhin sind auch diese Spuren verschwunden. Die Zelle ist mit Zymogenkörnern stark gefüllt, ihre Ausdehnung hat ihr Maximum erreicht.

Der Kern ist blass tingirt, wenig hervortretend. Die Zellen sind jetzt zur Funktion reif, tritt diese ein, so schrumpfen sie wieder zusammen auf das anfangs beschriebene Volumen. Diese Betheiligung an der Sekretion macht es erklärlich, dass man den Nebenkern bei längere Zeit hungernden Thieren, wie auch schon Nussbaum ermittelt hat, selten trifft, dass er sich am zahl- 
reichsten nach einer vorangegangenen guten Fitterung findet. Freilich sind nicht alle Alveoli auf dem gleichen Stadium zu treffen, sie zeigen vielmehr ein recht wechselndes Verhalten.

In den Malphighi'schen Gefässen lässt sich der Nebenkern schlecht verfolgen; da sein Uebergang unter die aus der Zelle austretenden grossen Sekretkugeln seine Auffindung sehr erschwert. Ich hoffe aber an Schnittpräparaten auch hier in sein Verhalten noch näher eindringen zu können. Von den in diesen Organen vorkommenden direkten Theilungen mit ungleichen Hälften, die sich wenn auch selten finden, ist der Sprossungsprozess, der den Nebenkern liefert, leicht zu trennen. Die Theilungsprodukte der Kerndurchschnürung sind, abgesehen von der Grösse und der dieser entsprechenden Nucleolenzahl, einander völlig gleich in Tinktionsfähigkeit und äusserm Habitus, dabei stets abgerundet, nie lappig oder gezackt, fallen ausserdem immer in die Pause nach der Sekretion, wo die Regeneration auftritt.

Im Pankreas des Salamanders findet der Ersatz der zn Grunde gehenden Zellen durch reguläre Mitosen statt, die ich oft in vorzüglich konservirtem Zustande vorfand.

Einen Punkt möchte ich der Vollständigkeit halber hier noch kurz erörtern. Bei der starken Inanspruchnahme der Zellen ist es erklärlich, dass sie rasch abgenutzt werden und dann absterben. Es weisen hierauf auch die zahlreich sich findenden Mitosen hin, welche einen Ersatz schaffen. Ich musste demnach auch darauf gefasst sein, regressiven Metamorphosen der Zellen zu begegnen. Dies ist in der That der Fall und sie sind interessant genug, um ihnen, abgesehen davon, dass ich diesem Thema ein besonderes Kapitel widmen werde, hier einen kleinen Platz zu gönnen.

Flemming hat den regressiven Kernveränderungen den Namen "Chromatolyse" gegeben und ihrer auch in seiner letzten Abhandlung wieder erwähnt (4).

Der Prozess fibrt zunächst zur Bildung meist kugeliger grösserer und kleinerer Elemente, die zunächst sich noch färben, später aber die Aufnahme des Safranins verweigern. Dieser Schwund der Tinktionsfähigkeit ist nun ein zunächst lokalbeschränkter und äusserst wechselvolle bizarre Figuren hervorbringender Prozess. Eine Auzahl derselben habe ich in den beigefügten Figuren wiedergegeben. Wer nach mehr verlangt findet 
bei Lukjanow (9) eine vollständigere Aufzählung und Beschreibung derselben.

Höchst anffallender Weise kann dieser regressive Prozess znnächst nur einen Theil des Kerns befallen, was ich als partielle Chromatolyse bezeichnen möehte. Flemming (3) nennt die jetzt zu beschreibenden Elemente: „tingible Körper". Der Kern \%eigt von scharfen Rändern begrenzte, wie mit einem Locheisen ausgeschlagene und der Membran zunächst entbehrende Liicken, in welchen die kugeligen Elemente der Chromatolyse liegen. Weiterhin kann wieder eine Abrundung des Kerns und Neubildung sciner Membran stattfinden. $O b$ er sich aber auf die Dauer erhalten kann, möchte ich stark bezweifeln. In wie weit ferner an der Bildung der in Rede stehenden eigenthümlichen Elemente eingewanderte weisse Blutkörperchen einen Antheil haben, konnte ich nicht feststellen. Heidenhain (8) hat neuerdings iiber derartige Fälle in den Darmepithelien des Salamanders berichtet. Derselbe Autor hat ebenfalls die höchst eigenthümlichen Bilder beschrieben, welche die Gegenwart eines Parasiten, eines Coccidiums in den Kernen hervorruft. Ueber die von $\mathrm{Gaule}$ (7) beobachteten Hümatozoën, die dieser den Nebenkernen einreihte und Cytozoën nannte, haben die Arbeiten Danilewsky's (1) Aufschluss gebracht.

Was nun noch schliesslich das Auswandern des Kernkörperchens aus dem Kern anlangt, wie es 0 gata (12) beschreibt, so babe ich dergleichen Bilder auch erbalten, aber merkwürdiger Weise nur an Paraffinschnitten, der bekannten Methode Gaule's. Bei Celloidineinbettung fehlten sie. Als ich später die Ovarien von Aulostomum in Paraffin schnitt, babe ich sehr hänfig den Keimfleck durch eine Liicke der Kernmembran ausgetreten gesehen, mich aber auch überzengt, dass ihn das Messer herausgerissen batte und das Gleiche gilt auch von dem obigen Befund. Derselbe ist also ein Artefakt.

Dass die Produkte der Chromatolyse, die ich hier nur vorläufig abgehandelt habe, natürlich bei ihrer weitern Veränderung gegen Farbstoffe in der verschiedensten Weise reagiren, dürfte leicht verständlich sein; deshalb aber in ihnen Dinge besonderer Art erkennen zu wollen und mit besondern Namen zu belegen, wie es Lukjanow gethan hat, halte ich für verfehlt und kann mich daher anch nicht für die von diesem Autor verwandte Färbung begeistern. In wie weit die Chromatolyse bei der Sekretion 
eine Rolle spielt, lässt sich noch nicht entscheiden. Im Pankreas dürfte sie bei ibrem spärlichen Vorkommen dies nur in untergeordnetem Maasse thun.

Zum Schlusse möchte ich noch bemerken, dass in den Details meiner Untersuchungen wohl Vieles wird nachzutrager sein. Für mich hat es sich zunächst nur darum gehandelt, iiber die an der Zelle und ihrem Kern wahrnehmbaren Veränderungen eine Uebersicht zu gewinnen. Dabei mussten hauptsächlich die Geschlechtszellen und die Drüsenzellen in Betracht kommen; da bei den andern Geweben die Verhältnisse weit einfacher liegen. Mit den genannten Elementen habe ich mich daher auch bisher am eingehendsten befasst und wünsche nichts mehr, als dass sich möglichst bald Jemand finden möge, der die gewonnenen Resultate einer Prüfung werth erachtet. Ich habe mich bemüht, iberall die günstigsten Objekte ausfindig zu machen und die Metbode zu einer möglichst sichern zu gestalten. Möge man nicht vergessen, dass die Pionierarbeit in einem neuentdeckten Gebiet eine miihselige ist. Der Nachuntersucher hat es weit leichter.

\section{Literatur.}

1) Danilewsky, B., Die Hämatozoën der Kaltblüter. Arch. f. mikrosk. Anat. Bd. XXIV, p. 588-099. Taf. XXVII A. 1885.

2) D a vid of f, M. v., Untersuchungen über die Beziehungen des Darmepithels zum lymphoiden Gewebe. Arch. f. mikrosk. Anat. Bd. XXIX, p. 495 bis 525, Taf. XXX und XXXI. 1887.

3) F l e m m ing, W., Studien über Regeneration der Gewebe. I. Arch. f. mikrosk. Anat. Bd. XXIV. p. 50-92. Taf. IV. 1885.

4) Fle mming, W., Neue Beiträge zur Kenntniss der Zelle. Arch. f. mikrosk. Anat. Bd. XXIX. p. 389-464. Taf. XXIII-XXVI. 1887.

5) Frenzel, J., Ueber den Darmkanal der Crustaceen nebst Bemerkungen zur Epithelregeneration. Arch. f. mikrosk. Anat. Bd. XXV. p. 137-191. Taf. VIII und IX. 1885.

6) Frenzel, J., Einiges über den Mitteldarm der Insekten, sowie über Epitheiregeneration. Arch. f. mikrosk. Anat. Bd. XXVI. p. 229-307. Taf. VII-IX. 1886.

7) G a u l e, Kerne, Nebenkerne und Cytozoën. Centralblatt f. d. medic. Wissenschaften. Nr. 31. 1881. 
8) Heidenha in, Beiträge zur Histologie und Physiologie der Dünn darmschleimhaut. Pf lü g e r's Arch. f. d. ges. Physiologie. Bd. XLIII. Supplementheft. 1888.

9) Lukjanow, S. M., Beiträge zur Morphologie der Zelle. Arch. f. Anat. und Phys. Suppl.-Bd. p. 66-90. 1887.

10) N u s s b a m, Sitzungsberichte der niederrh. Gesellschaft für Naturund Heilkunde. p. 183. 1881.

11) Nussbaum, Ueber den Bau und die Thätigkeit der Drïsen. Arch. f. mikrosk. Anat. Bd. XXI. p. 343. 1882.

12) Ogata, Masanori, Die Veränderungen der Pankreaszellen bei der Sekretion. Arch. f. Anat. und Physiol. Phys. Abth. p. 405-437. 1883.

13) P latner, G., Ueber die Entstehung des Nebenkerns und seine Beziehung zur Kerntheilung. Arch. f. mikrosk. Anat. Bd. XXVI. p. 343369. Taf. XIV. 1886.

14) Pl a t n e r, G., Mittheilungen zur histologisehen Technik. Zeitschr. f. wiss. Mikrosk. etc. Bd. IV. p. 349-452. 1887.

\title{
V. Samenbildung und Zelltheilung im Hoden der Schmetterlinge.
}

\author{
Hierzu Tafel XIII.
}

Die vorliegende Mittheilung über die Spermatogenese bei den Lepidopteren kann nicht beanspruchen, dieses Thema erschöpfend zu behandeln. Die Resultate wurden ausschliesslich an Schnitten gewonnen und bediirfen noch der Ergänzung durch Beobachtungen an Dissociationspräparaten. Die principiell wichtigen Punkte, auf deren Feststellung es mir am meisten ankam, lassen sich jedoch durch erstere Methode mit genügender Vollständigkeit eruiren. Es liess sich dreierlei als sicher nachweisen: erstlich, dass das Centrosoma zum Spitzentheil des Spermatosoms wird; sodann, dass der Rest des Kopfes lediglich aus dem Chromatin des Kerns der Spermatide hervorgeht; endlich, dass der aus der Substanz der Spindelfasern bervorgehende Nebenkern zar Umhüllung des Axenfadens verwandt wird.

Beror ich auf die Details näher eingehe, möchte ich zur besseren Orientirung einige allgemeine Bemerkungen vorausschicken. Ich habe mich bei meinen Untersuchungen mebr und mehr davon 
tiberzeugt, dass bestimmte Generationen der samenbildenden Zellen sich durch charakteristische Merkmale sowobl in ihrer Form, als auch besonders in dem Modus ibrer Theilung streng von den übrigen unterscheiden. Die von v. la Valette $S$ t. George eingeführte sehr brauchbare und auch allgemein angenommene Nomenklatur liefert eine genügende Anzahl von Bezeichnungen, die ich nur an bestimmte Generationen der Zellen gekniipft wissen möchte und zwar nach folgendem Schema:

1) Letzte Zellgeneration: Spermatiden, Samenausbildungszellen,

2) vorletzte Zellgeneration Spermatocyten II. Ordnung. drittletzte $\quad\left\{\begin{array}{l}\text { Samenvermehrungszellen I. Ord- } \\ \text { nung. }\end{array}\right.$

3) frühere Zellgenerationen: Spermatogonien. Stammsamenzellen.

Die Spermatocyten entsprechen den Eiern. Die zwei Theilungen, welche sie noch eingehen, denen der beiden Richtungsspindeln. In bejden Fallen findet eine Reduktion der chromatischen Substanz auf ein Viertel ihrer ursprünglichen Quantität statt, indem die zweite Theilung ohne eingeschaltetes Ruhestadium sofort an die vorhergehende sich anschliesst. Was nun die Kennzeichen anlangt, welche die Spermatocyten von den Spermatogonien zu unterscheiden gestatten, so sind dies folgende. Zunächst zeichnen sich die Spermatocyten durch ihre beträchtliche Grösse aus, besonders ist auch noch das Protoplasma im Verhältniss zum Kern sehr vermehrt. Diese Differenzen sind bei den von mir untersuchten Lepidopteren so bedeutende, dass sie allein schon genügen, um die betreffende Zellart sofort erkenuen za lassen. Da bei diesen Thieren das Auftreten der grossen Spermatocyten mit dem Beginn der wärmeren Jahreszeit zusammenfällt, so könnte man hierin eine Ursache für dieses plötzliche starke Zellwachsthum erblicken, indessen müssten dann alle Spermatogonien zugleich diese Veränderung eingehen, was, wie ich fruher gezeigt habe, nicht der Fall ist. Als bedingendes Moment kann man immerhin das Steigen der Teuperatur gelten lassen. Bei den Pulmonaten findet der Vebergang mehr allmählich statt. Die Unterscheidung der einzelnen Zellgenerationen wird dadurch eine viel schwierigere. Hier muss ein anderes Merkmal bei der Beurtheilung mehr berticksichtigt werden. Es ist dies die Differenz in dem Theilungsmodus der Spermatocyten und Spermatogonien. Bei den Lepidopteren lässt 
sich ohne Mühe bei der Theilung der letzteren Zellen das Vorkommen regulärer Knäuel und Schleifen feststellen, die, wie meine frtihern Untersuchungen ergeben haben, bei der Theilung der Spermatocyten vôllig fehlen.

Es ist mir wie Prenant (11) völlig unmöglich gewesen, die in der Zwitterdruise der Pulmonaten sich findenden Theilungsphasen in eine einzige Reihe zu bringen. Mit Leichtigkeit gelingt dieses aber, wenn man mit Rücksicht auf die leicht festzustellenden Verhältnisse bei den Lepidopteren den Spermatogonien einen andern Theilungsmodus zuschreibt als den Spermatocyten. Man kommt damit zur Aufstellung zweier verschiedener Theilungsreihen, wie dies auch Prenant schon gethan hat. Hat man auf diesem Wege aber erst einmal den ersten Schritt gethan, so gelingt es leicht, auch noch weitere Merkmale aufzufinden, welche die Berechtigung einer solchen Scheidung dokumentiren.

Ich kann mich hier noch nicht näher darauf einlassen, diese Verhältnisse eingehender zu behandeln, sondern werde in einer speziellen Abhandlung darauf zurỉckkommen. Hier mag es genigen zu betonen, dass Spermatogonien und Spermatocyten wesentliche Differenzen auch bei den Pulmonaten zeigen und in dieser Beziehung eine weitgehende Uebereinstimmung zwischen diesen Thieren und den Lepidopteren herrscht.

Von grosser Bedeutung ist mir ferner der Umstand erschienen, dass die Prophasen der Spermatocytentheilung bei den Lepidopteren eine auffallende Uebereinstimmung zeigen rait den Phänomenen, welche die Bildung der ersten Richtungsspindel bei Aulastomum gulo begleiten. Einige Punkte lassen sich bier noch genauer feststellen, so z. B. der Verbleib der Kernmembran.

Ebenso wie die 'Theilung der Spermatocyten ist die der Richtungsspindeln ausgezeichnet durch den Mangel eines typischen Kernknäuels sowie der Chromatinschleifen. Es treten nur kurze Stäbchen auf bei den meisten bisher hierauf näher untersuchten Objecten oder doch eine starke Reduktion der Schleifen. Einen Unterschied möchte ich indessen nicht zu erwähnen vergessen. Während die beiden Theilungen der Spermatocyten stets senkrecht auf einander erfolgen, geschehen die der beiden Richtungsspindeln in derselben Richtung. Es mag dies vielleicht nur in der besondern Grösse der Eier und der Anordnung des Dottermaterials seinen Grund baben, was ich für das Wahrscheinlichste halte, indessen könnte diescm 
Umstand doch auch noch eine tieferer Bedeutung inne wohnen. Da die erste Richtungsspindel bei Aulastomum an jedem Pol ein schönes Centrosoma trägt (Fig. 1, Taf. XIII), so wäre es interessant zu erfahren, wo das Centrosoma am pheripheren Pol der zweiten Richtungsspindel herrührt, oder sollte sich etwa hier dann keines finden? Ich hoffe, dass die Fortsetzung meiner Untersuchungen bei Hirudineen den erwünschten Aufschluss bringen wird. Nocb andere wichtige Fragen harren der Entscheidung; so ist das Verhalten des vom Eikern und des vom Spermakern herrührenden Centrosomas näher zu erforschen, worauf auch Rabl (12) neuerdings aufmerksam gemacht hat. Bleiben beide Elemente isolirt oder findet eine Vereinigung derselben statt?

Eine andere Frage durfte schon jetzt zu entscheiden sein. Diese betrifft die Bedeutung der Richtungskörperchen. Der Zweck ihrer Bildung ist die Hervorbringung eines kopulationsfähigen Kerns, also eines Halbkerns. Um dieses zu erreichen, werden zwei Theilungen sich unmittelbar einander folgen müssen. Eine einzige 'Theilung würde zwei Kerne liefern, die zwar zunächst an Masse nur halb so gross wären als der Mutterkern, im Uebrigen aber diesem völlig gleichwerthig sein würden. Werden dieselben aber nun sofort noch einmal getheilt, so hat jeder der jetzt entstehenden Kerne den Werth nur eines halben Kerns, was sich auch darin zeigt, dass die Zahl der chromatischen Elemente um die Hälfte vermindert ist. Die erste Theilung hat daher nur den Werth eines vorbereitenden, aber damit der Zweck erreicht wird, unbelingt nothwendigen Aktes. Die unabweisbare Consequenz hiervon ist nun, dass, wenn die zweite Theilung der Spermatocyten der der zweiten Richtungsspindel entspricht, die erste Theilung derselben der der ersten Richtungsspindel homolog ist. Alles was demnach für die Theilungsprodukte dieser letzteren gilt, muss auch auf die aus der ersten Theilung der Spermatocyten hervorgehenden Zellen angewendet werden können. Ich möchte nun wissen, was daraus werden sollte, wenn in die Kerne der einen Spermatocyte zweiter Ordnung nur das histogene, in die Kerne der andern das Keimplasma übergehen wollte, wie es der Theorie Weismanns (13) entsprechen würde.

Wie kommt es nun, dass, während bei der Spermatogenese alle aus der Theilung einer Spermatocyte erster Ordnung hervorgehenden Zellen respektive Kerne benutzt werden, bei den Thei- 
lungen der Richtungsspindeln nur einer der entstehenden Kerne zur Verwendung gelangt, die andern aber einfach geopfert werden.

Ich muss hier wieder etwas vorgreifen und erwähnen, dass das Ei bei Bildung der ersten Richtungsspindel nur das nakte Centrosoma entbält ohne jede Spur von Archoplasma. Ein solches bildet sich erst bei der Entstehung der Polstrablungen um jedes der Centrosoma. Nun weiss man aber aus den Untersuchungen von Boveri (3), dass Centrosoma und Archoplasma für sich allein ohne Kern eine Zelltheilung bewirken können. Der Mangel an genïgendem Archoplasma vermag vielleicht die ungleiche Theilung des Protoplasmas bei der Richtungskörperchenbildung zu erklären. Sodann mag das Princip der Natur, ein Individuum möglichst bald vermehrungsfähig zu machen, dazu geführt haben, im Ei nur das Material für die Entstehung eines Embryo anzuhäufen. Die Eier miissten, wenn jeder Richtungskern eine entsprechende Dottermenge erhielt, viermal so gross sein, und es bleibt fraglich, ob sie dann noch theilungsfähig wären, wie man in der That bei den dotterreichen Eiern der Insekten keine Zelltheilung auf die Kerntheilung folgen sieht.

Wäre dieser Prozess aber an den Beginn der Eireife gesetzt, so würde nichts den Kern hindern können, ebenso wie das Protoplasma sich vermehrt, zu der ursprünglichen Grösse eines theilungsreifen Kerns wieder heranzuwachsen. Alles was von dem Richtungskörperchenproblem noch übrig bleibt, wïrde demnach die Frage sein, wie wird es möglich, dass hier, was sonst nie geschieht, die Ruhestadien zwischen zwei Kerntheilungen ausfallen; dasselbe gilt es natürlich anch für die Theiluug der Spermatocyten zu erforschen.

Der Dimorphismus in der Zelltheilung im Hoden ist eine schon öfter beobachtete Thatsache. Ausser den Angaben Carnoy's (4) und meinen frühern (10) liegt auch eine ausfubrliche Mittheilung von Flemming (5) hierüber für Salamandra vor, auf welche ich gelegentlich noch näher einzugehen habe.

Ich habe nun zunächst die besten von meinen Lepidopterenpräparaten mit den mir jetzt zu Gebote stebenden guten Systemen nochmals einer genauen Prtfung unterzogen. $\mathrm{Da}$ ist es mir denn olne Mühe gelungen, in den Spermatocyten das Centrosoma an der Zellspitze an dem Punkt, nach welchem die Protoplasmastränge konvergiren, wie ich dies früher beschrieben habe, zu finden. Es 
liegt bier in der ruhenden Zelle ein runder, stark lichtbrechender Körper, umgeben von einem hellen Hof, der wieder von einer Reihe dunkler Körnchen eingefasst ist. Ich schlage vor, diesem ganzen Gebilde den Namen Nebenkern, mit dem es sein Entdecker v. la Valette $\mathbf{S t}$. George einst belegt hat, auch ferner zu lassen. Man wird unter dieser Bezeichnung daher zu verstehen haben das Centrosoma sammt seiner Umgebung, soweit sie sich an der Spindelbildung betheitigt.

Ein solcher Nebenkern findet sich nun auch in den Eiern und zwar von den frühsten Entwickelungsstadien an. Ja er tritt in den letzteren am deutlichsten hervor, wie ich mich bei Aulastomum iiberzeugen konnte (Fig. 2). Sobald erst einmal im Protoplasma die spezifischen Dotterelemente auftreten, wird es unendlich schwer, ihn sicher zu verfolgen. Man ist gar zu leicht Täuschungen ausgesetzt, and es hat mir grosse Mühe gekostet, seine Theilung und Betheiligung an der Spindelbildung, die in den wegen ibres sehr feinkörnigen Dotters noch am besten sich hierzu eignenden Eiern von Aulastomum in genau derselben Weise erfolgt, wie in den Spermatocyten der Lepidopteren, sicher festzustellen. Ich babe den Nebenkern bei den ersten Entwicklungsstadien der Eier von Pulmonaten beschrieben und abgebildet. Die Bezeichnung des hier gefundenen Elementes als Nebenkern nach Analogie des in den samenbildenden Zellen vorkommenden Körpers war, wie die Folge gezeigt hat, eine durchaus korrekte. Ich muss mich demnach sehr entschieden gegen die Unterstellung Garnauld's (6), als habe ich Dinge beschrieben, die gar nicht existiren, verwahren. Dieser Forscher wird sich ebenso wie meine belgischen Gegner wohl noch an die Existenz des Nebenkerns gewöhnen miussen. Eine Vergrösserung des Nebenkerns habe ich während des Wachsthums der Eier nicht konstatiren können. Dass ich jetzt iubrigens über den Ursprung dieses Elements ganz andre Ansichten hege wie früher, brauche ich wohl nicht $z u$ betonen. Solange seine Bedeutung noch ganz in Dunkel gehtillt war, konute man über die Entstehung den versehiedensten Auffassungen huldigen. Die ausgezeichneten Untersuchungen $\nabla$ a n Beneden's (1) sowie Boveri's (3) lassen keine andere Annahme mehr zu, als dass man es hier mit einem jeder Zelle von der ersten Furchung an zukommenden Element zu thun hat, das nur einer nicht immer gleichen weitern Ausbildung unterliegt, so dass es besonders in den samen- 
bildenden Zellen eine bedeutende Entwicklung erlangt und daher hier auch zuerst beobachtet wurde. Die später im Pankreas aufgefundenen Elemente haben eine ganz andre Bedeutung and wïrde es daher sich empfehlen, auch eine andere Bezeichnung für dieselben zu wählen. Vielleicht dürfte "Zynoblast" passend sein.

Um zu den Spermatocyten zurückzukehren, so bemerkt man, bevor noch im Kern irgend welche wahrnehmbaren Veränderungen auftreten, eine Streckung des Centrosomas, welche der umgebende Hof mit macht. Hieran schliesst sich die Theilung an, so dass man zwei Centrosomas nebeneinander in dem länglichen Hofe erhält (Fig. 4). Die dunklen peripheren Körnchen, die zuweilen auch als kurze Stäbchen erscheinen, häufen sich hierbei etwas stärker an den beiden äussersten Enden der hellen Aureole an. Endlich theilt sich auch die letztere. Die beiden auf diese Weise entstandenen Gebilde rücken weiter aus einander und nähern sich dem Kern (Fig. 5), an dem sie scbliesslich als zwei knopfförmige Aufsätze, wie ich sie früher beschrieben habe, erscheinen. Im Anschluss hieran tritt auch immer deutlicher die Anordnung der Protoplasmastrahlen zu polaren Astern hervor. Was die weiteren Vorgänge anlangt, in Bezug auf welche ich nur für die Beschaffenheit der Pole meinen frühern Augaben einige Ergünzungen zuzufitgen habe, so kann ich hier um so eher darliber hinwegrgehen, als ich dieselben Phänomene bei Besprechung der Bildung der Richtungsspindel in den Aulastomumeiern zu beschreiben haben werde. Die einzige Abweichung wird durch das eigentbümliche Verhalten des Keimflecks bedingt, der erst sebr spät sicb allmählich auflöst.

$\mathrm{Zu}$ dem Resultate, dass die gesammte Zelle auf das Centrosoma centrirt ist, ist nun auch $\mathrm{Rabl}$ (12) auf Grund theoretischer Schlussfolgerungen gelangt, so dass dieselbe Thatsache zwei Forscher völlig unabbängig von einander, der eine auf Grund direkter Beobachtung, der andere infolge sorgfältiger Erwägungen, fast zu derselben Zeit gefunden haben, gewiss ein schöner Beweis für die Richtigkeit und Bedeutung derselben. Wer meine friher gegebenen Abbildungen kennt (10; Fig. 1 und 3), welche deutlich das Zusammenlaufen der Protoplasmastrahlen in einem Punkt zeigen, wird sich tiberhaupt wundern miissen, wie ich das an dieser Stelle liegende und jetzt auch mit voller Deutlichkeit dort erkannte Centrosoma habe ubersehen können (Fig. 3). 
Spermatogenese.

Pygaera bucephala und Sphinx Euphorbiae sind die Objekte, bei welchen die nachfolgenden Beobachtungen angestellt wurden. Gegen Ende der letzten Spermatocytentheilung bildet sich um die nahe den Polen gelegene chromatische Substanz ein heller Hof (Fig. 6). An der polaren Seite desselben befindet sich das noch von einer deutlichen Strahlung umgebene Centrosoma. An der aequatorialen Seite liegen eine Anzahl runder Körner von wechselnder Grösse, aus den benachbarten also polaren Enden der Spindelfasern hervorgegangen. Der Rest der Spindelfasern persistirt noch, wandelt sich aber weiterhiu in einen runden, undeutlich granulirten Körper um, ebenso sammeln sich die erwähnten Körner an einer Stelle an und verschmelzen zu einem soliden Element (Fig. 7). Die beiden soeben beschriebenen Gebilde rerdanken also den Spindelfasern ihren Ursprung und zwar das grosse dem aequatorialen, das kleine dem polaren Abschnitt derselben. Die Spermatide enthält ausser ibnen noch das Centrosoma, um welches die Asterstrahlung sich inzwischen zurückgebildet hat, sowie die Kernvakuole. In letzterer hat das anfangs noch plattenförmig angeordnete Cbromatin sich inzwischen zu Körnern aufgelösst, welche sich an die Peripherie begeben, wo eine deutliche Kernmembran jetzt auftritt (Fig. 8). Ich schlage nun vor, da der Name Nebenkern entschieden für den in den Spermatocyten sich findenden Körper geeigneter erscheint, für die den Spindelfasern entstammenden Körper einen andren Namen, etwa „Mitosoma“, wodurch zugleich ein Hinweis auf seine oft fädige Struktur gegeben wäre, zu wählen. Ich werde mich wenigstens, um jede Verwirrung zu vermeiden, einstweilen desselben bedienen.

Um das grosse Mitosoma, welches einen undeutlich granulirten oder mehr fädigen Bau zeigt und an dessen Bildung sich wohl auch das Protoplasma mehr oder weniger betheiligen mag, bildet sich weiterhin ein oft breiterer, oft nur schmaler heller Saum aus, es auf diese Weise scharf gegen die übrige Zellsubstanz abgrenzend. Etwa auf dem gleichen Stadium bemerkt man im Innern desselben die ersten Spuren des Centralfadens des Spermatosoms, von der Kernmembran ausgehend die Mitte des grossen Mitosomas und weiterhin auch das Protoplasma durchsetzend, welches bereits eine starke Verlängerung zeigt (Fig. 8).

Die Lage der Spermatiden in den Alveolen hat sich dahin 
verändert, dass, während früher die Kerne dem Lumen zugewandt waren, sie jetzt nach der Alveolenwand hin in den Zellen gelagert sind, so dass deren Protoplasmafortsätze, welche die Umbildung zum Spermatosom einleiten, in den Hohlraum der Alveolen sich erstrecken. Die einzelnen Bestandtheile einer Spermatide für sich betrachtet zeigen folgendes Verhalten. Der Kern enthält die chromatische Substanz nur an der Peripherie vertheilt und der Membran angelagert, so dass sie auf Längs- wie auf Querschnitten in der Form eines an einzelnen Stellen durchbrochenen Ringes erscheint (Fig. 8 und 9). Der Innenraum des Kerns zeigt nur den homogenen Kernsaft.

Das Centrosoma liegt der Kernwandung dicht an, bald an dem oberen Theil, das heisst, der dem Ansatz des Axenfadens entgegengesetzten Seite derselben, bald mebr seitlich. In den meisten Fällen wird es dem Beschauer auf der hellen Innenfläche des Kerns deutlich entgegentreten (Fig. 10). Geeignete Schnitte (Fig. 8 und 9) zeigen aber, dass es draussen liegt. Das kleine homogene Mitosoma liegt gleichfalls dem Kern dicht an, an seiner untern Seite nabe dem Ansatzpunkt des Axenfadens an der Kernwandung. Das grosse Mitosoma wird in seiner Mitte vom Axenfaden durchsetzt und tritt an der Stelle, wo dieser inserirt, gleichfalls dicht an den Kern heran. Die Protoplasmastränge zeigen im allgemeinen noch eine Richtung parallel dem Längsdurchmesser der Zelle, entsprechend ibrer Anordnung bei der letzten Theilung. Die nächsten Veränderangen, welche eintreten besteben darin, dass das grosse Mitosoma eine Verlängerung eingeht, wobei es sich mehr und mehr an dem Axenfaden berunterzieht, ihn so mit einer Hiulle umkleidend. Der helle Saum, welcher dasselbe anfangs umgab, wird dabei immer schmäler und entzieht sich auf diese Weise schliesslich völlig der Wahrnehmung (Fig. 11). Eine entsprechende Verlängerung des Protoplasmas muss natürlich vorausgegangen sein. Es hält aber schwer, liber ibre Ausdehnnng eine bestimmte Anschauang za gewinnen, da die Schnitte ein Spermatosom in seiner ganzen Ausdehnung nicht za verfolgen gestatten. Ein weiterer Fortschritt in der Umbildung vollzieht sich dadurch, dass die chromatische Substanz, welche bisher an der ganzen Peripherie des Kerns vertheilt war, sich nach einem Punkt derselben koncentrirt (Fig. 12). Die Kernmembran selbst verfällt weiterhin der Auflösung. Indessen markirt sich der helle Kernraum selbst noch lange mit 
scharfer Grenze gegen die Umgebung. In denselben geräth nun das Centrosoma hinein und tritt zu der chromatischen Substanz in bestimmte Beziebung. Das Chromatin bildet nämlich einen balbkugeligen oder noch frther schalenförmigen Körper (Fig. 12). In der Mitte der äussern konvexen Seite desselben inserirt sich der Axenfaden. In der Mitte der konkaven oder planen, nach dem Kernraum gewendeten Seite nimmt das Centrosoma seine definitive Lage an. Während der chromatische Körper sich mehr und mehr in der Richtung des verlängerten Axenfadens streckt und dabei aus der halbkugeligen allmählich mehr in eine kugelige, weiterhin ovale Form tibergeht (Fig. 14-17), hat sich auch das kleine Mitosoma aktiv betheiligt. Es nimmt seine definitive Stellung an der Stelle, wo der Axenfaden an den chromatischen Kopf inserirt, ein, dann ändert es seine ovale Form in eine mehr spindelförmige oder, da es der Krummung der Peripherie des Kernraums hierbei folgt, besser gesagt, halbmondförmige um, so dass der ganze vordere Abschnitt des sich bildenden Spermatosoms in seinem Bereich jetzt eine mebr oder weniger ausgedehnte Krümmung zeigt, die mit der weiteren Streckung des kleinen Mitosomas noch zunimmt; nacbdem dieser Prozess aber sein Maximum erreicht hat, sich wieder ausgleicht. Dass bierbei der Axenfaden von dem kleinen Mitosoma nur umkleidet, nicht aber etwa vom Kopf getrennt wird, erseheint wohl zweifellos. Es gelang mir freilich nicht mit Sicherheit, denselben innerbalb der Substanz des Mitosomas nachzuweisen, was aber wohl an frischen Dissoeiationspräparaten nicht schwierig sein duirfte. Das Spermatosom zeigt jetzt einen ovalen oder birnförmigen Kopf, der lediglich ans Chromatin besteht und nur an seinem vordern Ende das z. Th. etwas in ihn eingesenkte Centrosoma trägt (Fig. 17). Der Faden des Spermatosoms enthält den Axenfaden sowie eine Umbällung, welche von den Mitosomas (Nebenkern), also in letzter Linie von der Substanz der Spindelfasern berrührt. -Inwieweit noch das Protoplasma dabei betheiligt ist, lässt sich nicht feststellen. Der Kopf braucht sich jetzt nur noch etwas zu verlängern und zuzuspitzen, um seine definitive Form anzunehmen. Das fertige Spermatosom dürfte wohl am besten an frischen Präparaten zu untersuchen sein, was ich bei der näehsten Gelegenheit nachzubolen nicht verabsäumen werde. Für das Verständniss der nach dem Eindringen in das Ei an dem Spermatosom sich abspielenden Veränderungen genügen die mitgetheilten Beobachtungen. 
Lediglich zu diesem Zweek habe ich dieselben auch hier erörtert, da meine Untersuchungen uber die erste Entwickelung befruchteter und parthenogenetischer Eier von Liparis dispar, uber die ich schon vorläufig berichtet habe, bald in diesem Archiv in ausführlicher Darstellung erscheinen sollen.

Die Mittheilungen, welche Gils on (7) über die Spermatogenese bei den Lepidopteren macht, enthalten von den soeben geschilderten Details fast nichts, so dass ich keine Veranlassung habe, hier näher auf dieselben einzugehen.

Eine weitgehende Uebereinstimmung mit den von v. la Valette St. George (8) bei andern Arthropoden erhaltenen Resultaten lissst sich nicht verkennen.

Das Hervorgehen des Nebenkerns (Mitosoma) aus den Spindelfasern, die Betheiligung desselben an der Umbüllung des Axenfadens sind von diesem Forscher schon bei andern Insekten festgestellte Thatsachen. Dass im vorliegenden Falle zwei Nebenkerne (Mitosoma) auftreten, dürfte wohl nur in der besondern Beschaffenheit der achromatischen Spindel begrundet sein.

Eine Vermuthung möchte ich hier noch äussern. Es hat mir oft den Anschein gehabt, als ob auch der Axenfaden nicht sowohl ein Produkt des Zellprotoplasmas sei, als viehmehr gleichfalls irgend welcben Residuen der Spindelfasern entstamme; leider bin ich noch zu keinem positiven Ergebniss in dieser Beziehung gelangt.

Im allgemeinen kann man wohl sagen, ein Spermatosom entspricht der Anordnung seiner Substanzen nach einer Tochterzelle gegen:Schluss der Theilung. Es befindet sich nämlich in beiden Fällen an der Spitze das Centrosoma, auf dasselbe folgt das Chromatin und hieran schliesst sich die Substanz der Spindelfasern. Nur ist bei dem Spermatosom alles stark in die Länge gezogen. In dieser Form dringt dasselbe in das Ei ein. Die Ansicht Nussbaum's (9), dass es sich bei der Befruchtung um die Vereinigung homologer Zellen handle, hat demnach entschieden mehr für sich, als das entgegengesetzte Extrem, nämlich einzig dem Kern hierbei eine Rolle zuzuschreiben. Ein spezifischer Zellbestandtheil wird jedenfalls allem andern voran dem Eikörper einverleibt, das ist das Centrosoma. Es löst sich im Ei wieder vom Kopf des Samenfadens los und ist bei Liparis von einer freilich nur wenig hervortretenden Strahlung umgeben. Damit stimmen auch die Angaben verschiedener Beobachter, welche die um den Spermakern sich 
bildende Strahlenfigur nicht auf diesen selbst, sondern auf einen neben denselben gelegenen Punkt orientirt sein lassen, wie dies nenerdings wieder A. A. Böhm (2) fiù das Ei von Petromyzon beschrieben hat.

\section{Literatur.}

1) Van Beneden et A. Neyt, Nouvelles recherches sur la fécondation ct la division mitosique chez l'Ascaride mégalocéphale. Bulletin de l'Acad. royale de Belgique, 3me Série T. XIV. Nr. 8, 1887.

2) Bö hm, A. A., Ueber Reifung und Befruchtung des Eies von Petromyzon Planeri. Arch. f. mikrosk. Anat. Bd. XXXII. p. 613-671. 1888.

3) Boveri, Th., Zellstudien II. Die Befruchtung und Theilung des Eies von Ascaris megalocephala. Jenaische Zeitschr. f. Naturw. Bd. XXII. 1888.

4) Carnoy, J. B., La Cytodierèse chez les arthropodes. La Cellule. P. I. 1884.

5) Flemming, W., Neue Beiträge zur Kenntniss der Zelle. Arch. f. mikrosk. Anat. Bd. XXIX. p. 389-464. 1887.

6) Garnauld, P., Sur les phénomènes de la fécondation chez l'Helix aspersa et l'Arion empiricorum. Communication préliminaire. Zool. Anz. Nr. 296-298, 1888, 1889.

7) Gilson, Étude comparée de la Spermatogénèse chez les arthropodes. La Cellule. T. I. 1884.

8) v. la Valette St. George, Spermatologische Beiträge. Zweite Mittheilung. Arch. f. mikroskop. Anat. Bd. XXVII. p. 1-13. Taf. I u. II. 1886.

9) Nussbaum, M., Ueber die Veränderungen der Geschlechtsprodukte bis zur Eifurchung. Ein Beitrag zur Lehre von der Vererbung. Arch. f. mikrosk. Anat. Bd. XXIII. p. 155-214. 1884.

10) Platier, G., Die Karyokinese bei den Lepidopteren als Grundlage für eine Theorie der Zelltheilung. Internat. Monatsschr für Anat. und Hist. Bd. III. Heft X. 1886.

11) Prenant, A., Observations cytologiques sur les éléments séminaux des Gastéropodes pulmonés. La Cellule. T. IV. I. fase. 1888.

12) Rabl, C., Ueber Zelltheilung. Briefliche Mittheilung an Herrn Geheimrath von Kölliker. Mit 2 Abb. Anat. Anz. IV. Jahrg. Nr. 1. 1889.

13) Weismann, A., Die Kontinuität des Keimplasmas als Grundlage einer Theorie der Vererbung. Jena 1885. 


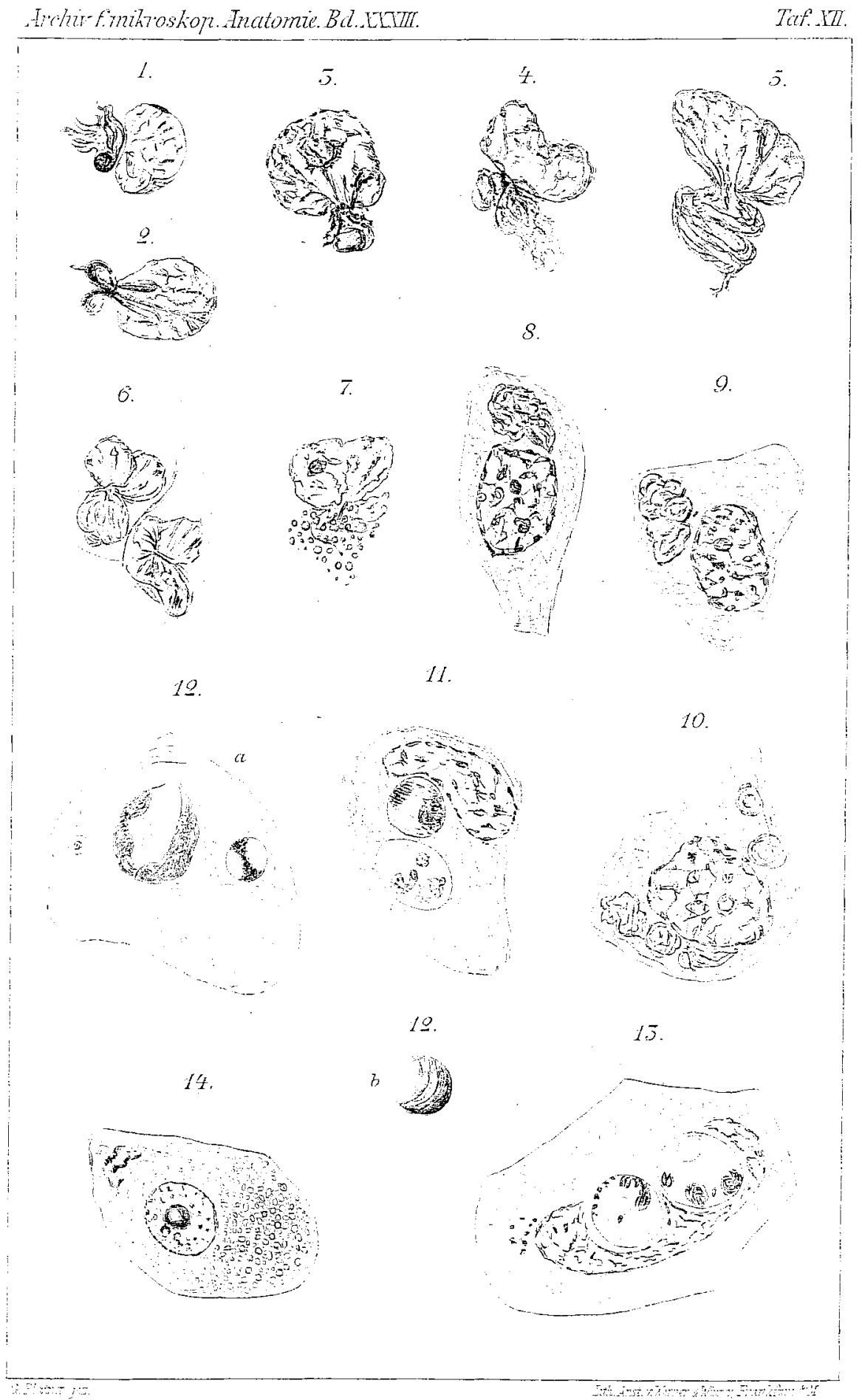




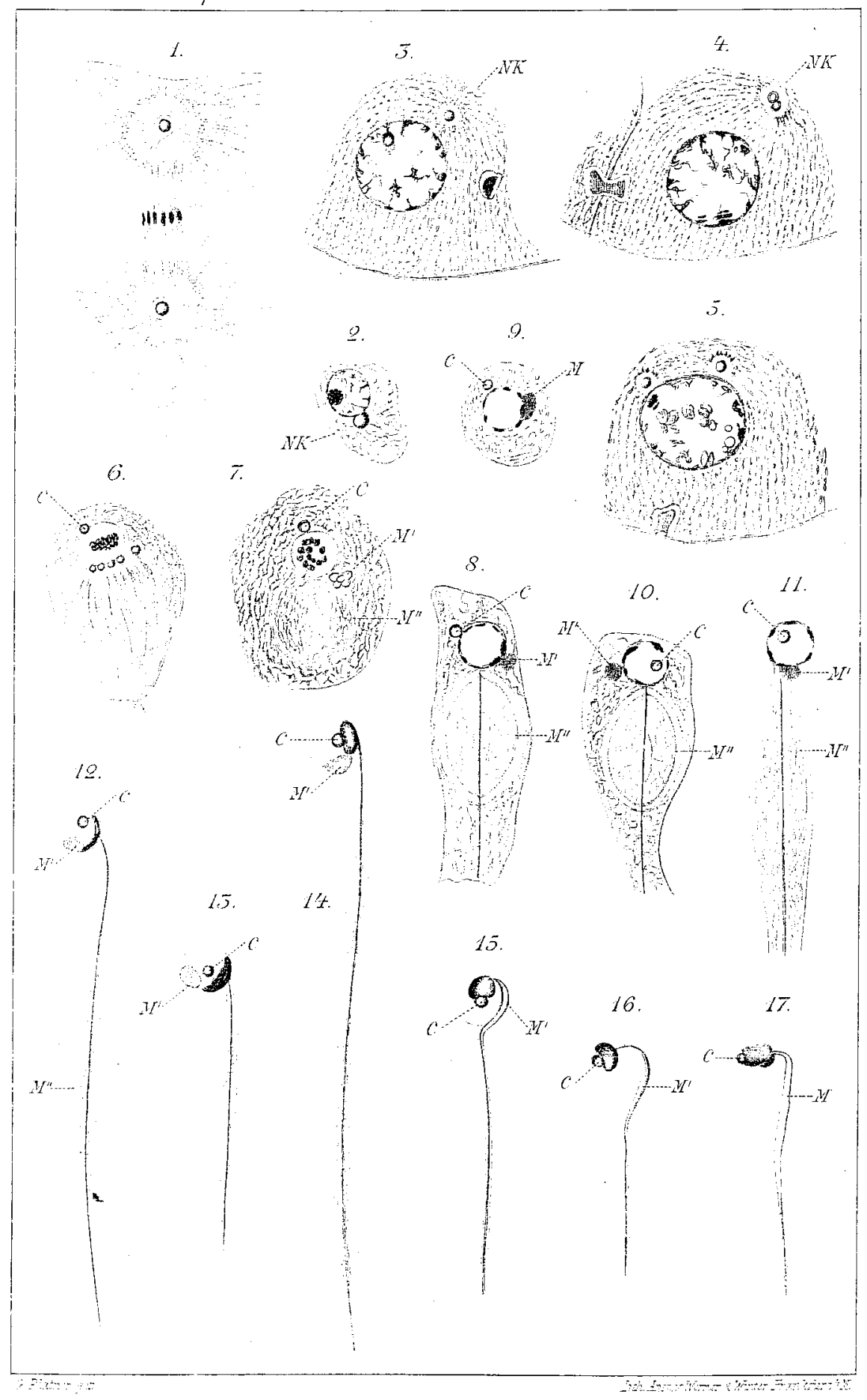

